\title{
Computational study of velocity distribution and pressure drop for designing some gas quench chamber and furnace ducts
}

\author{
O. Macchion ${ }^{\text {a }}$, N. Lior ${ }^{b, *}$, A. Rizzi ${ }^{\mathrm{c}}$ \\ a Faxénlaboratoriet, Department of Mechanics, Royal Institute of Technology, Sweden \\ ${ }^{\mathrm{b}}$ Department of Mechanical Engineering and Applied Mechanics, University of Pennsylvania, USA \\ ${ }^{\mathrm{c}}$ Department of Aeronautical and Vehicle Engineering, Royal Institute of Technology, Sweden
}

\begin{abstract}
Gas cooled quenching and many other applications require high-speed uniform-velocity flows, with minimal pressure drop. The flow ducting geometry is often rather complex, with flow splitting, $90-180^{\circ}$ bends, and circular-to-rectangular cross-section transition ducts (the latter are used, for example, between the circular blower duct and the rectangular quenching baskets). Similar situations exist in forced convection furnaces. To provide design guidance in the choice of such ducts, and focusing primarily on circular-to-rectangular transition ducts, the flow was modelled and computed, and the results were successfully validated. Sensitivity of the velocity uniformity and pressure drop with respect to the primary geometric parameters, pressure, and Reynolds numbers was examined in the range (1.3) $10^{5}$ $\# \operatorname{Re} \#(7.8) 10^{5}$, with an ultimate objective to produce optimal designs. For a length-to-diameter ratio $A L=L / D<1.0$, flow nonuniformity at the exit plane and pressure drop are increased by 33 and $83 \%$, respectively, as the aspect ratio (rectangular duct width-to-height) $A R$ decreases from 4 to 1 . Increasing $A R$ beyond 1.5 leads to linearly increasing nonuniformity and pressure drops. A diverging-contracting duct has proven to lead to lesser nonuniformity, while it did not influence the pressure drop. Increasing the inlet pressure from 1 to 20 bar led to a decrease in flow distortion by $11 \%$ at the duct exit planes. At atmospheric pressure, increasing the Reynolds number from (1.3) $10^{5}$ to $(7.8) 10^{5}$ increased distortion by $8 \%$. Some preliminary design recommendations for circular-to-rectangular duct transitions are to try and keep $A L>1$ and $A R<1.5$.
\end{abstract}

(C) 2004 Elsevier B.V. All rights reserved.

Keywords: Circular-to-rectangular duct transitions; Duct flow; Duct bends; Quench chamber design; Velocity uniformity; Furnace design

\section{Introduction}

Circular-to-rectangular transition ducts are essential components of gas quench chambers, aero engines, and ventilation devices. As part of a long-term R\&D program at the Faxénlaboratoriet to advance gas-cooled heat treatment, it was shown in [1] how flow homogeneity influences the hardness uniformity of gas-quenched metal parts. Flows in circular-to-rectangular transition ducts of constant cross-sectional area have been addressed by several experimental and computational studies with different objectives and performance criteria. Miau et al. [2] experimentally investigated three circular-to-rectangular ducts with length-to-diameter ratios of $1.08,0.92$, and 0.54 , under low subsonic flow conditions at $R e_{\mathrm{D}}=(1.96) 10^{5},(6.77) 10^{5}$, (1.1) $10^{6}$. The exit aspect ratio $A R$ was equal to two and the cross-sectional area was constant for all three ducts. Mean

\footnotetext{
* Corresponding author. Tel.: +1 215898 4803; fax: +1 2155736334 . E-mail addresses: olivier@mech.kth.se (O. Macchion), lior@seas.upenn.edu (N. Lior), rizzi@kth.se (A. Rizzi).
}

flow and turbulence data were taken at the inlet and exit planes. Secondary flow patterns indicative of streamwise vortex formation were observed at the exit lane of the ducts. From these results, all the terms in the axial mean direction were computed. Their analysis showed that the generation of streamwise vorticity is due primarily to transverse pressure gradients induced by geometrical deformation.

Burley and Carlson [3] investigated the effect of circular-to-rectangular transition duct aspect ratio, length and shape on thrust ratio, and total pressure losses at $R e_{\mathrm{D}}$ $=(5.4) 10^{6}$ and high subsonic flow conditions. Five different circular-to-rectangular transition duct configurations were investigated to explore the effects of duct length, wall shape, and cross-sectional area distribution on performance. The transition ducts were installed in a transonic wind-tunnel with a high exit aspect ratio $A R$, nonaxisymmetric nozzle downstream of the transition duct, and the overall internal performance was measured. In addition, one duct was tested with swirl vanes installed. Discharge coefficient and thrust ratio versus nozzle pressure ratio were used as performance criteria. The results of their investigation show that for 


\begin{tabular}{|ll|}
\hline \multicolumn{2}{|l}{ Nomenclature } \\
$a$ & width of the exit rectangular duct \\
$A$ & cross sectional area of duct \\
$A L=L / D$ & duct length ratio \\
$b$ & duct aspect ratio \\
$D$ & height of the rectangular duct \\
$K$ & duct inlet diameter \\
$L$ & pressure drop coefficient \\
$N$ & length of transition duct \\
$P$ & flow nonuniformity as defined in Eq. (3) \\
$P t$ & static pressure \\
$q 0$ & total pressure \\
$r$ & dynamic pressure \\
$R e_{\mathrm{D}}$ & radius at the corners of the rectangular \\
$=\rho U D / \mu$ & cross-section \\
$R 1$ & Reynolds number based on inlet \\
& diameter and inlet velocity \\
& semi-major axis of the ellipse defining \\
$R 2$ & the geometry of the bend presented in \\
& Section 4 \\
& semi-minor axis of the ellipse defining \\
$S_{\text {inlet }}$ & the geometry of the bend presented in \\
$T$ & Section 4 \\
$U$ & $=\pi(D / 2)^{2}$, duct inlet area \\
$x, X, y, Y, z, Z$ & temperature \\
$\delta$ & inlet velocity \\
$\eta$ & duct dimensional coordinates \\
$\mu$ & boundary layer thickness \\
& exponent of the superelliptic cross- \\
section & dynamic viscosity \\
& fluid density \\
&
\end{tabular}

length ratios $A L$ less than or equal to 0.75 , large regions of separated flow are present. Swirling the flow had a positive effect on performance.

The review of the literature has revealed that there is only a small amount of experimental or computational data on circular-to-rectangular transition ducts of constant cross-sectional area distribution. Moreover, the flow conditions that have been investigated address often transonic conditions [3] when the study is intended for gas-turbine improvement or only a limited range of parameters [2] when the intention is to increase the knowledge in fluid dynamics. No handbook data (cf. [4-6]) were found on the topic. The present computational sensitivity analysis of the geometrical parameters and of the flow conditions influencing velocity uniformity and pressure drop in circular-to-rectangular transition ducts is intended to help fill this void. All computations were run for air at ambient temperature, $T=298.3 \mathrm{~K}$. The computations that did not aim at testing the influence of pressure were run at $P=1 \mathrm{bar}$. Influence of length was tested by varying the length aspect ratio $A L$ between 0.7 and 1.5. Influence of $A L$ was shown by computing the flow in ducts whose exit $A R$ was varied from 1.0 to 4.0 (square shape to rather flat rectangle). Comparison of flows in an expanding-contracting duct and a constant cross-sectional area duct was performed. Influence of pressure $(1,6$, and 20 bar) and Reynolds number in the range (1.3) $10^{5}$ \# Re \# (7.8) $10^{5}\left(10,20,30\right.$, and $60 \mathrm{~m} \mathrm{~s}^{-1}$ at the inlet plane) were studied.

\section{Computational model}

\subsection{Transition duct model configurations}

Flows in several transition ducts have been studied. The transition ducts' cross-sectional shapes were designed by using a sequence of superelliptic cross sections

$$
\left(\frac{y}{a}\right)^{\eta}+\left(\frac{z}{b}\right)^{\eta}=1
$$

where $a$ and $b$ are, respectively, the semi-major axis and semi-minor axis of the superellipse (see Fig. 1), $\eta$ is the exponent. Circles with $a=b$ and $\eta=2$, and rectangles with $\eta \rightarrow \infty$ are included in this family of curves. The inlet diameter of each transition duct was $D=0.20428 \mathrm{~m}$ (dimensions of the experimental duct), as the duct used in [5] was taken as a reference for most computations.

The maximum height and width of the duct cross-section were represented by cubic functions which were chosen to achieve continuity of the second derivative at the end points of the ducts. These functions defined $a$ and $b$ of the superellipse. The exponent of the superellipse was calculated from an implicit function relating the quantities $a, b$, and $\eta$ to the area $A$ of the superellipse

$A=\frac{\Gamma(1 / \eta)^{2}}{\Gamma(1 / \eta)}(2 / \eta) \cdot(4 a b)$

where $\Gamma(\cdot)$ denotes the gamma function. $A R=a / b$ and $A L$ $=L / D$ were the exit aspect ratio of the rectangular duct and the length ratio of the transition duct, respectively. As seen in Eq. (2), the relationship between $a, b$, and $\eta$ determines also the radius at the corners of the rectangular cross-section by setting the value of $\eta$. Following the lead of [7], it was chosen to have $\eta=10$ for all geometries. This value corresponds to a radius of $r=0.015 \mathrm{~m}$ at the corners of the rectangular cross-section. We also note that the magnitude of $r$ has some influence on the flow distribution and pressure drop, with larger $r$ tending to improve uniformity and reduce pressure drop, but this has not been examined in this study.

\subsection{Solver and boundary conditions}

The three-dimensional coupled fully implicit NavierStokes code, CFX 5.5.1, high-resolution scheme [2] was used for flow analysis. Five turbulence models $(k-\omega$ of Wilcox, SST of Menter [6], SSG Reynolds stress model of Speziale et al. [9], QI and RSM of Launder et al. [10]) were 


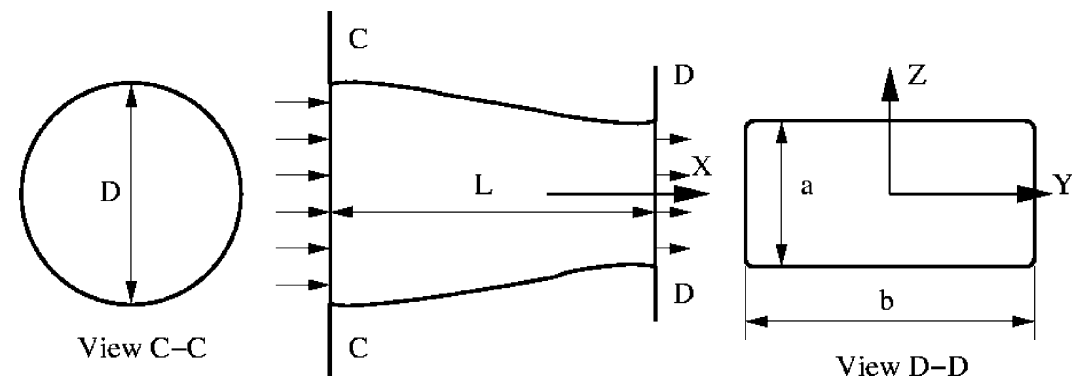

Fig. 1. Streamwise velocity contours produced by the computational code and compared against the experimental results of [8] $(P=1 \mathrm{bar}, T=298.3 \mathrm{~K}$, $\left.R e_{\mathrm{D}}=(5.9) 10^{5}\right)$.

tested and the results were compared with the experimental measurements presented in [5]. The three Reynolds stress models that were tested gave similar results and underestimated the strength of the secondary motion that develops in the transition duct by $20 \%$. Compared to the results given by the eddy-viscosity models, the Reynolds stress models did not give any better results. It is interesting to notice that several researchers $([11,12])$ have used the test case [7] as a benchmark for turbulence model testing in 3D flows. Sotiropoulos and Patel $[11,12]$ have shown that good predictions could be achieved using a near-wall second moment closure, but the results concerning the streamwise velocity contours are not much better than the ones got with the present model. Extensive testing in [13] has shown good agreement between computed and experimental wall pressures at different stations along the duct centerline. Moreover, it was shown that the main features of the flow field (a pair of counter-rotating vortices originating from change in cross-section) have indeed been reproduced by the computation. Fig. 2 represents the comparison between streamwise velocity contours produced by the computational code and the experimental results of [7]. This agreement was considered sufficient for engineering purposes as the maximum error was less than $20 \%$. As the Reynolds stress models do use wall functions and thus do not need a fine resolution of the near-wall layer, the Reynolds stress models were

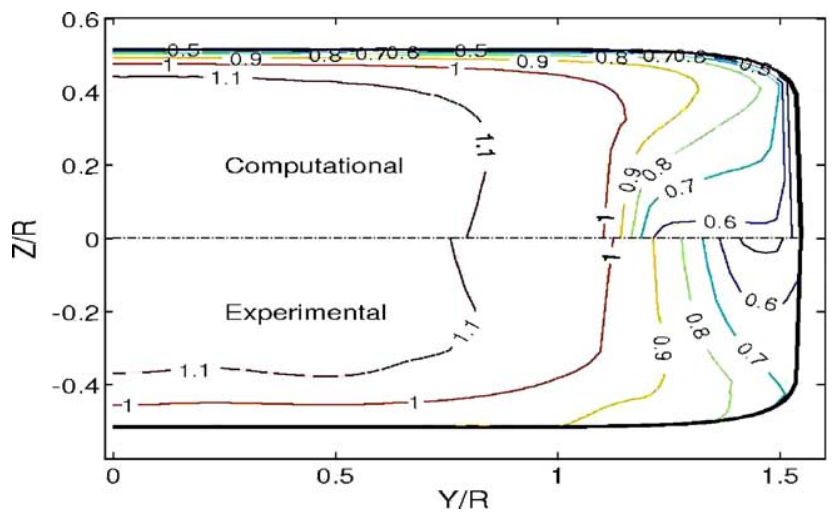

Fig. 2. Relation between nonuniformity $N$ and the streamwise velocity profile on a line taken at the intersection of the symmetry plane $\mathrm{XZ}$ and the exit plane of a circular-to-rectangular transition duct for $A L=1.05$, $P=1$ bar, $T=298.3 \mathrm{~K}, R e_{\mathrm{D}}=(5.9) 10^{5}$. preferred to the expensive near-wall eddy-viscosity models. All calculations presented in this paper were performed using the QI model of Launder et al. [10] described in [14].

Only one quadrant of the duct was represented in the grid domain. Therefore, mirror image symmetry conditions for the flow-field were imposed at the quadrant boundaries. The inlet profile for all computations was the experimental semi-developed turbulent profile specified in [7]. Turbulent intensity at the inlet was $0.3 \%$. Grid sensitivity tests indicated that a hybrid mesh with 1,357,315 elements is sufficient to obtain grid-independent solutions (see [14] for details).

The flow conditions for the computational analysis were chosen to correspond to the experiments reported in [7], where the transition duct was tested at atmospheric pressure.

\subsection{Performance criteria}

Velocity nonuniformity at the exit of the transition duct, over its cross-sectional area, was evaluated using the criterion $N$, defined as

$N=\frac{1}{u_{\mathrm{avg}}} \sqrt{\iint_{S} \frac{1}{S}\left(u-u_{\mathrm{avg}}\right)^{2} \mathrm{~d} S}$

where $u_{\max }$ and $u_{\text {avg }}$ are, respectively, the maximal and averaged mean streamwise-direction velocity in the duct cross-section. Fig. 3 represents the relation between nonuniformity values $(N)$ and the streamwise velocity profile taken at the intersection of the exit plane and the symmetry plane $\mathrm{XZ}$ (see Fig. 1). For $N<0.17$, the velocity profile is similar to a classic turbulent boundary layer velocity profile over a flat plate without separation. However, for $N>0.17$, the velocity profile shows the influence of the secondary motion due to the curvature of the walls of the transition duct. To evaluate the relationship of the practical velocity uniformity to $N$, the data presented in Fig. 2 were extrapolated to higher $N$ values. The conclusion was that these types of duct geometries, having a constant cross-sectional area and exhibiting no flow separation, are unlikely to produce much higher flow nonuniformity $(N)$ values. This could be checked by a series of computations where the exit aspect ratio is continually increased until separation is reached. This conclusion is confirmed by another study performed by us for a duct which 


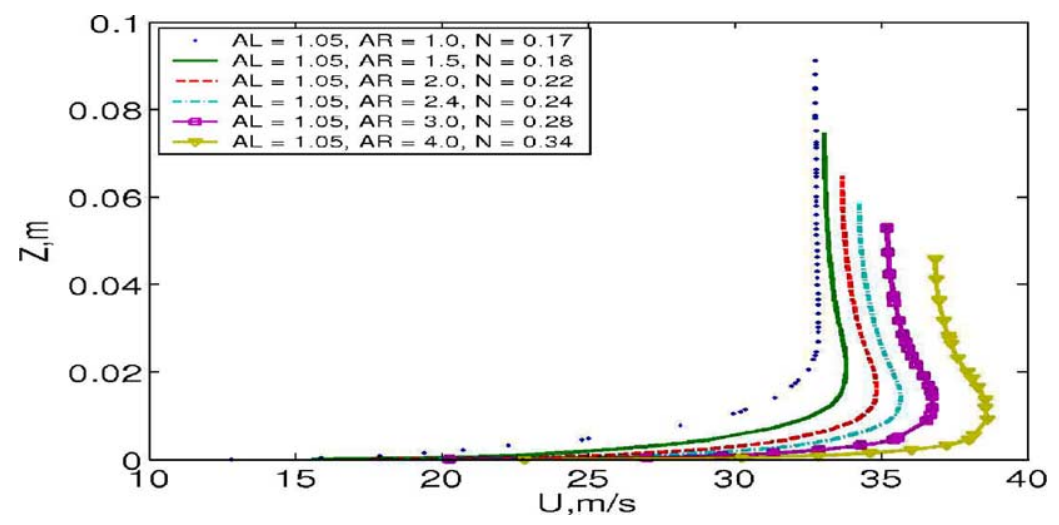

Fig. 3. Example of the geometry of the circular-to-rectangular transition ducts that have been tested. Here, $A L=L / D=1.5, A R=a / b=2.0$.

starts with a 90 bend connecting to a circular-to-rectangular transition $\left(A R=2.0, A L=1.0, U=20 \mathrm{~m} \mathrm{~s}^{-1}, P=1 \mathrm{bar}, T\right.$ $=298 \mathrm{~K}$, with a ratio of the exit area to the inlet area of 2.0) that exhibits separated flow and leads to $N=1.32$, which is comparatively much higher than the $N=0.34$ obtained here for the unseparated flow within a circular-to-rectangular transition duct $(A L=1.05, A R=4.0$, same flow conditions). Other criteria (cf. [15]) are being investigated to get a better understanding of the characterization of flow nonuniformity.

Pressure drop coefficient $K \equiv \Delta P_{\mathrm{t}} / q_{0}$ estimation was based upon the values of total pressure at the exit $\left(P_{\mathrm{t}_{\mathrm{e}}}\right)$ and inlet $\left(P_{\mathrm{t}_{\mathrm{i}}}\right)$ planes of the transition ducts, with $\Delta P_{\mathrm{t}} \equiv P_{\mathrm{t}_{\mathrm{e}}}-$ $P_{\mathrm{t}_{\mathrm{i}}}$ and $q_{0}=0.5 \rho U^{2}$ is the inlet dynamic pressure.

\section{Circular-to-rectangular transition duct}

\subsection{Duct length effects}

As shown in Fig. 4, increasing length leads to improved velocity uniformity (smaller $N$ ) for duct length ratios $A L$ $<1.0$, but has a much smaller effect for $A L>1$. It is also seen that $N$, as well as the effect of duct length, increases with the duct aspect ratio $A R$. This result was also noted in the experimental study in [3].

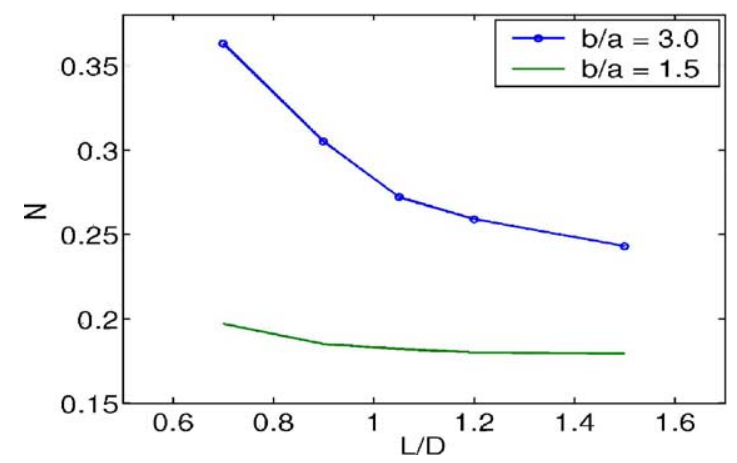

Fig. 4. Effect of transition length on velocity uniformity $(P=1 \mathrm{bar}, T$ $\left.=298.3 \mathrm{~K}, R e_{\mathrm{D}}=(5.9) 10^{5}\right)$.
Fig. 5 shows the influence of duct length on the total pressure drop. The conclusions drawn from the examination of Fig. 4 are somewhat similar here as well, showing a strong influence of increasing the length on reducing the pressure drop for $A R<1$, and a more moderate one for $A R>1$. It is noteworthy that total pressure losses arise from two different mechanisms - friction losses on the walls of the duct and losses due to transverse motion (secondary motion) in the flow field itself. Additional losses due to separated flow could be encountered. Nevertheless, no separation was seen to occur in any of the calculations that have been performed. This is attributed to the fact that no change in cross-section area was introduced.

Short transition ducts are known to lead to large pressure gradients and curvature effects, which in turn bring about large secondary motions. From Fig. 5, one can see that such transverse motion leads definitively to much larger losses than friction losses themselves.

\subsection{Duct exit aspect ratio $(A / R)$ effects}

As seen in Figs. 6 and 7, increasing aspect ratio leads to increased velocity nonuniformities and pressure drops. Analysis of the flow patterns at the exit plane show that increasing the aspect ratio leads to a strengthening of the streamwise vortex pair that develop in a circular-to-rectangular transition

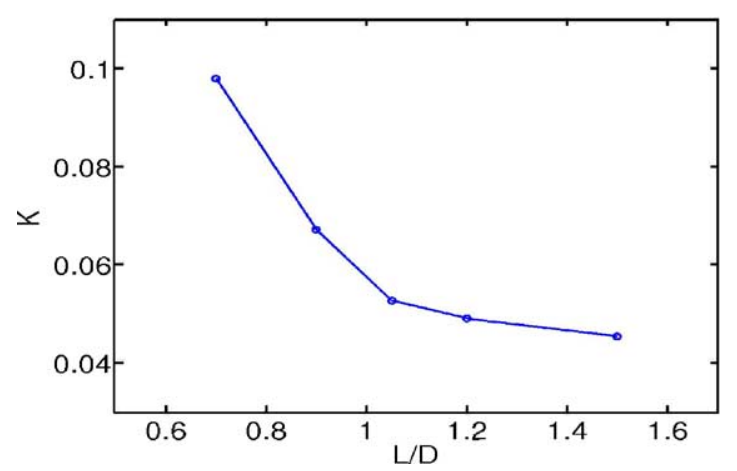

Fig. 5. Effect of transition length on pressure drop $(P=1 \mathrm{bar}, T=298.3 \mathrm{~K}$, $\left.\operatorname{Re}_{\mathrm{D}}=(5.9) 10^{5}\right)$. 


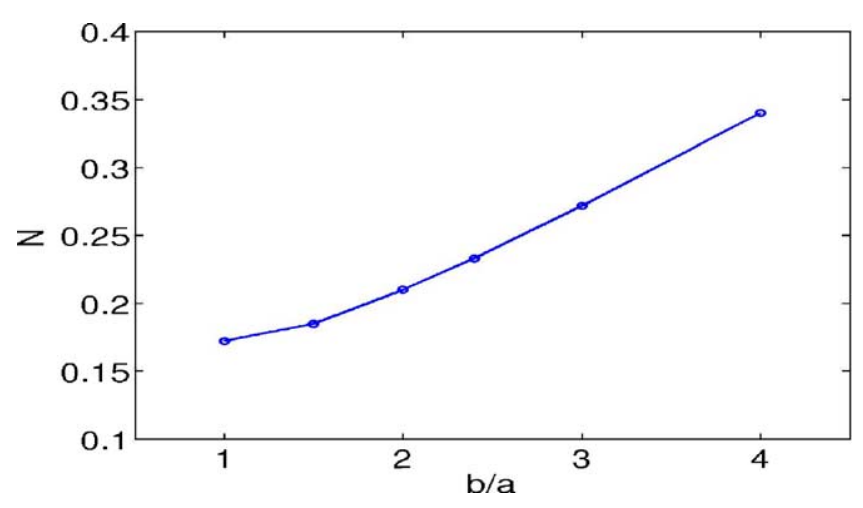

Fig. 6. Influence of exit aspect ratio $A R$ on nonuniformity for $A L=1.05$, $P=1$ bar, $T=298.3 \mathrm{~K}, R e_{\mathrm{D}}=(5.9) 10^{5}$.

duct. These induce the flow to move from the side walls toward the core flow along the semi-major axis of the superelliptic cross-section ([2,7]), and increase boundary layer mixing (i.e. when the two boundary layers cross each other), this decreasing the size of the primary motion area, all leading to the observed increased pressure drop.

\subsection{Expanding-contracting duct}

Computation of the flow through an expanding-contracting duct and a constant cross-sectional area duct have shown that the expanding contracting transition duct gives a flow whose distortion is reduced by $25 \%$ in comparison with the constant cross-sectional area duct, while no noticeable change was seen in the pressure drop. Both computations were performed for the same Reynolds number based on inlet diameter, $R e=(3.9) 10^{5}$. Both ducts had a length ratio $A L=L / D=1.5$ and an exit aspect ratio (AR) $=3.0$. Fig. 8 shows that the largest cross-sectional area is reached approximately at half-length of the transition duct.

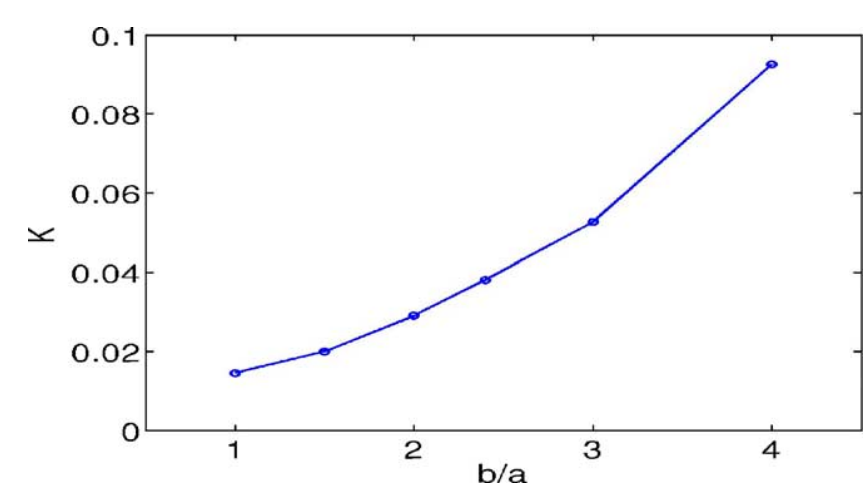

Fig. 7. Influence of exit aspect ratio $A R$ on pressure drop for $A L=1.05$, $P=1$ bar, $T=298.3 \mathrm{~K}, R e_{\mathrm{D}}=(5.9) 10^{5}$.

Burley and Carlson [3] had already noticed that a contraction helped to improve transition duct performance. This behaviour can be explained by the fact that a contraction has the effect of elongating the streamwise vortices that develop in the transition duct and consequently decrease the intensity of the vorticity.

Fig. 9 shows a plot of the transverse velocities at the exit plane of the transition duct. The cross-flow appears to be much stronger in the constant-area transition duct than in the expanding-contracting duct. The pair of counter-rotating vortices (see [7]) appears to be of less intensity in the constant area duct.

\subsection{Pressure effects}

Velocity uniformity increases with pressure, which can be related to the decrease of the boundary layer thickness, i.e. a decrease in the extent of the velocity regions which are slower than the core. Two facts have led to this interpretation. Firstly, at high pressure, the logarithmic layer was seen to be located much closer to the wall. The first grid point had to be located much closer to the wall during the grid generation

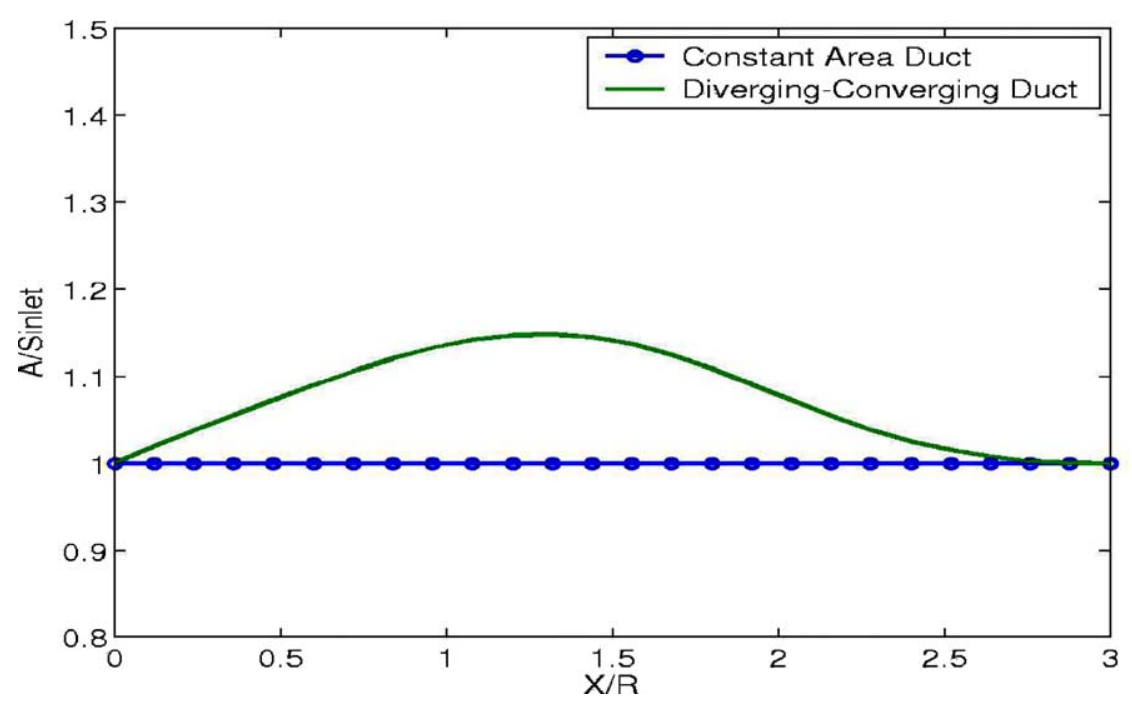

Fig. 8. Cross-sectional area ratio $A / S_{\text {inlet }}$ along the duct $(A L=1.5, A R=3.0)$. 


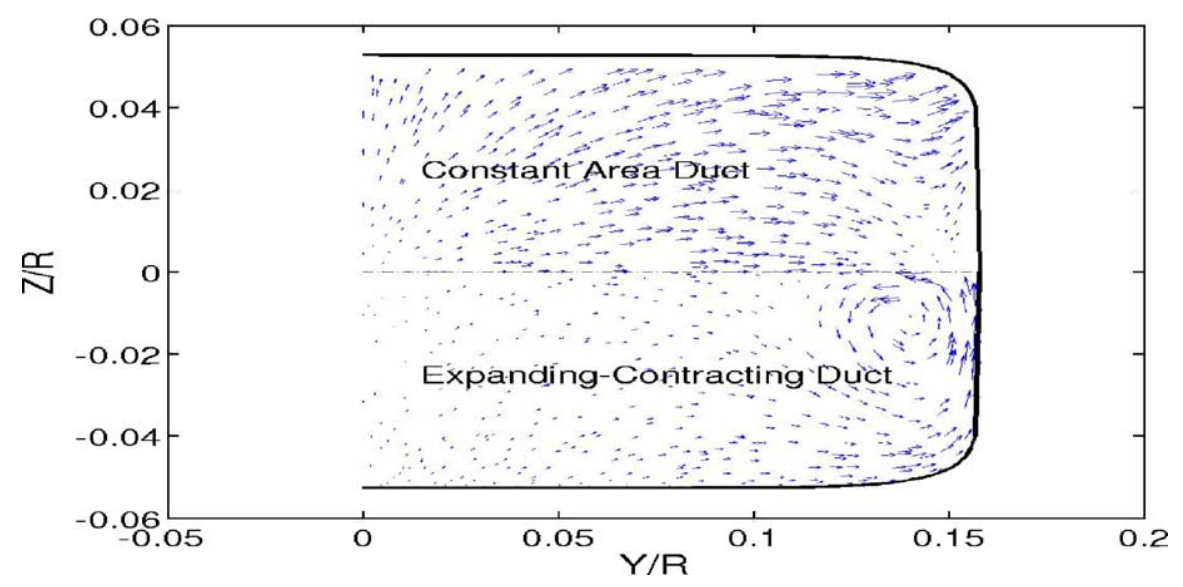

Fig. 9. Computed influence of an expanding-contracting transition duct on transverse velocities at the rectangular exit plane. Top half: constant area duct; bottom half: the expanding-contracting duct. $A L=1.5, A R=3.0, P=1 \mathrm{bar}, R e_{\mathrm{D}}=(3.9) 10^{5}, T=298.3 \mathrm{~K}$.

Table 1

Influence of pressure on velocity nonuniformity $N$ and boundary layer thickness $\delta(A L=1.05, A R=2.0)$

\begin{tabular}{ccll}
\hline$P$ (bar) & \multicolumn{1}{c}{$R e_{\mathrm{D}}$} & $N$ & $\delta(\mathrm{m})$ \\
\hline 1 & 390000 & 0.210 & 0.00330 \\
6 & 2340000 & 0.200 & 0.00300 \\
20 & 7800000 & 0.186 & 0.00260 \\
\hline
\end{tabular}

process to satisfy the criteria for grid generation presented in [14].

Secondly, as shown in Table 1, the boundary layer thickness measured at the exit plane was seen to decrease with increasing pressure.

\subsection{Reynolds number effects}

Table 2 shows a study of the influence of duct inlet bulk velocity on the flow properties at the exit plane of a transition duct with a length ratio $A L=0.9$ and exit aspect ratio $A R=2.0$. An interesting result is that while velocity nonuniformity gradually increases as the velocity is raised to about $30 \mathrm{~m} \mathrm{~s}^{-1}$, the nonuniformity decreases with a further velocity increase beyond a value between 30 and $60 \mathrm{~m} \mathrm{~s}^{-1}$. This behaviour has been previously noticed in the experimental study reported in [2], where it was seen that flow at $35.5 \mathrm{~m} \mathrm{~s}^{-1}$ was less distorted than flow at $6.1 \mathrm{~m} \mathrm{~s}^{-1}$. This

Table 2

Influence of duct inlet velocity on velocity nonuniformity $N$, boundary layer thickness $\delta$ and total pressure drop $\Delta P_{\mathrm{t}}(A L=1.05, A R=2.0)$

\begin{tabular}{lllll}
\hline$U_{\mathrm{b}}\left(\mathrm{m} \mathrm{s}^{-1}\right)$ & $R e$ & $N$ & $\delta(\mathrm{m})$ & $K$ \\
\hline 10 & 130000 & 0.296 & 0.00301 & 0.02 \\
20 & 260000 & 0.299 & 0.00297 & 0.02 \\
30 & 390000 & 0.312 & 0.00293 & 0.021 \\
60 & 780000 & 0.308 & 0.00276 & 0.021 \\
\hline
\end{tabular}

phenomenon was attributed in [2] to the fact that the flow is laminar at low velocities.

\section{4. $90^{\circ}$ duct bends \\ 4.1. Geometry and computational model}

The exit velocity nonuniformity and the overall pressure drop were computed for three types of $90^{\circ}$ duct bends. The first one was a constant radius bend with a fixed centre of curvature at $R 1=0.30 \mathrm{~m}$ of the centreline of the bend. The inlet diameter $D$ of the bend was $D=0.20 \mathrm{~m}$. The second configuration was similar to the preceding one, apart from the fact that the centerline was no longer a circular arc but an ellipse with a semi major axis $R I=0.45 \mathrm{~m}$ and semi minor axis $R 2=0.30 \mathrm{~m}$ (Fig. 10). In an attempt to examine whether expanding-contracting cross-section of a bend would decrease the nonuniformity and pressure drop, as we found it does for a constant cross-section duct, the third analyzed configuration has the same centreline shape as the second one, but the diameter of the duct increases to be $10 \%$ larger at mid-bend than at the inlet. Then, the bend contracts to reach an exit diameter equal to the inlet diameter. Those geometrical considerations are described in Fig. 10.

The same boundary conditions and turbulence model as those used in the above-described study of the transition ducts were applied here.

\subsection{Results}

Computational comparison of the flow nonuniformity and pressure drop between the above-described duct bends was carried out. The results are presented in Table 3 . Using a longer (elliptical) bend leads to reduction of the flow distortion by $27 \%$, and of the pressure drop by $7.5 \%$. The expansion-contraction analyzed here further 


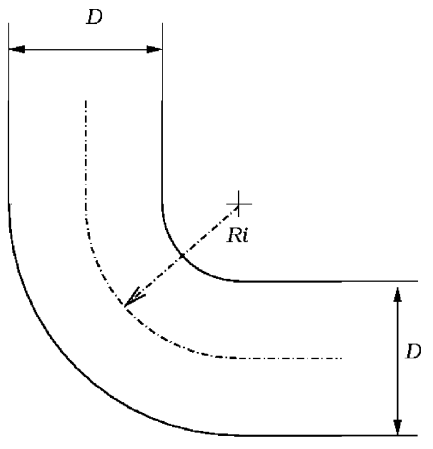

(a) Circular Centerline

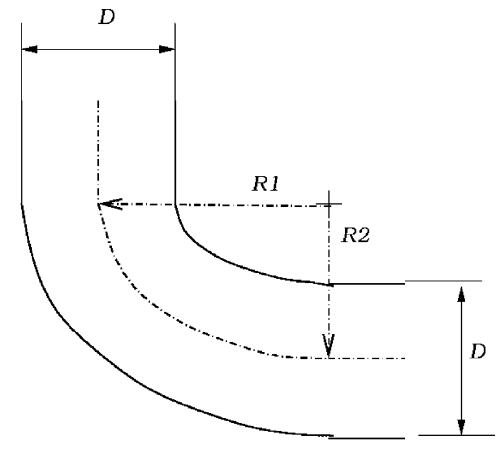

(b) Elliptical Centerline

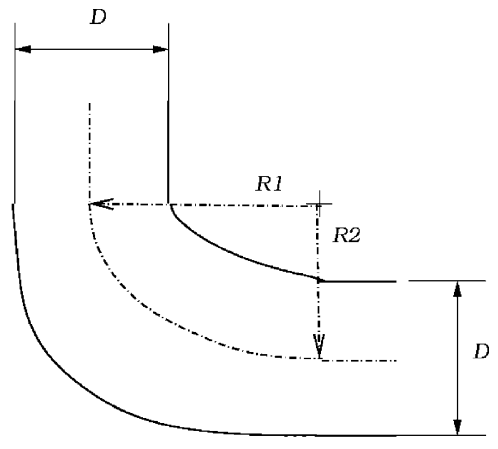

(c)
Elliptical Centerline - Expanding Contracting

Fig. 10. Geometrical features of the three bend configurations that were tested $\left(U=20 \mathrm{~m} \mathrm{~s}^{-1}, P=1\right.$ bar, $\left.\operatorname{Re}_{\mathrm{D}}=(3.9) 10^{5}, T=298.3 \mathrm{~K}\right)$.

Table 3

Influence of geometry on velocity nonuniformity $N$, and total pressure drop coefficient $K$, in three different duct bends: $P=1$ bar, $T=298.3 \mathrm{~K}$ and $R e_{\mathrm{D}}=(3.9) 10^{5}$

\begin{tabular}{lll}
\hline Geometry & $N$ & $K$ \\
\hline Circular centreline & 1.6 & 1.79 \\
Elliptical centreline & 1.4 & 1.66 \\
Expanding-contracting & 1.3 & 1.56 \\
\hline
\end{tabular}

reduces the flow distortion by $5 \%$ and the pressure drop by $6 \%$.

\section{Conclusions and recommendations}

Influence of geometry and flow parameters on the flow in circular-to-rectangular transition ducts have been studied. A Reynolds stress model has proven to be successful in the prediction of these flows. For $A L<1.0$, flow distortion at the exit plane and pressure drop are increased, respectively, by 33 and $83 \%$ as AR decreases. Exit $A R>1.5$ leads to linearly increasing distortion and pressure drops, the pressure drop being $K=0.02$ at $A R=1.5$, and $K=0.11$ at $A R=4.0$. A diverging-contracting duct has proven to lead to lesser distortion, while it did not influence the pressure drop. Increasing the inlet pressure from 1 to 20 bar led to a decrease in flow distortion by $11 \%$ at the duct exit planes. At atmospheric pressure, increasing the Reynolds number from $(1.3) 10^{5}$ to $(7.8) 10^{5}$ increased distortion by $8 \%$.

Some preliminary design recommendations for circular-torectangular duct transitions are to try and keep $A L>1$ and $A R<1.5$, and to use longer radii and perhaps expandingcontracting cross-sections in duct bends.

\section{Acknowledgements}

This work was performed by the Faxénlaboratoriet of the Kungl. Tekniska Högskolan (KTH), Stockholm, partially sponsored by LindeGas $\mathrm{GmbH}$ (formerly AGA AB), and
Ipsen International $\mathrm{GmbH}$. We also thank the CFX team at Harwell, and the people from CFX forum, for their good advice.

\section{References}

[1] A. Thuvander, A. Melander, M. Lind, N. Lior, F. Bark, Prediction of Convective Heat Transfer Coefficients and Examination of Their Effects on Distortion of Tubes Quenched by Gas Cooling, 4th ASM Heat Treatment and Surface Engineering Conference in Europe, Florence, Italy, 19-21 October, 1998.

[2] J.J. Miau, T.S. Leu, J.H. Chou, S.A. Lin, C.K. Lin, Flow distortion in a circular-to-rectangular transition duct, AIAA J. 28 (8) (1990) 1447-1456.

[3] J.R. Burley, II, J.R. Carlson, Circular-to-Rectangular Transition Ducts for High-Aspect Ratio Nonaxisymmetric Nozzles, AIAA-85-1346, NASA Langley Research Center, Hampton, VA.

[4] I.E. Idelchik, Handbook of Hydraulic Resistance, Hemisphere Publishing Corporation, 1986.

[5] D.S. Miller, Internal Flow Systems, Cranfield, BHRA, 1991.

[6] R.J. Manglik, Heat Transfer Fluid Flow Data Books, Genium Publishing Corporation, Amsterdam, 1998.

[7] D.O. Davis, F.B. Gessner, Experimental investigation of turbulent flows through a circular-to-rectangular transition duct, AIAA J. 30 (2) (1992) 367-375.

[8] F.R. Menter, Two-equation eddy-viscosity turbulence models for engineering applications, AIAA J. 32 (8) 1994.

[9] C.G. Speziale, S. Sarkar, T.B. Gatski, Modelling the pressure-strain correlation of turbulence: an invariant dynamical systems approach, J. Fluid Mech. 277 (1991) 245-272.

[10] B.E. Launder, G.J. Reece, W. Rodi, Progress in the developments of a Reynolds-stress turbulence closure, J. Fluid Mech. 68 (1975) 537566.

[11] F. Sotiropoulos, V.C. Patel, Prediction of turbulent flow through a transition duct using a second-moment closure, AIAA J. 32 (11) (1994) 2194-2204.

[12] F. Sotiropoulos, V.C. Patel, Turbulence anisotropy and near-wall modelling in predicting three-dimensional flows, AIAA J. 33 (3) (1995) 504-514.

[13] CFX Validation Report, Turbulent Flow Through a Circular-toRectangular Transition Duct, CFX-VAL07_0402, 12 April 2002. email: cfx-support-uk@ansys.com.

[14] AEAT, CFX 5 Solver and Solver Manager - Mathematical Models and Solution Algorithms, AEA Publishing, 2002.

[15] S. Lefantzi, D.D. Knight, Automated design optimization of a three-dimensional S-shaped subsonic diffuser, J. Propulsion Power, 18 (4) (2002) 